\title{
EFEITO DO EXERCÍCIO FÍSICO REGULAR NO EQUILIBRIO DE IDOSAS ATIVAS
}

Elaine Aparecida Lozano da Silva, Laís Alli Dias, Giovana Renata Parizi Silva, Vinícius Gustavo Gimenes Turato , Thayla Sayuri Suzuki Calderon, Alan José Barbosa Magalhães, , Cláudia Regina Sgobbi de Faria ,Regina Celi Trindade Camargo.

Universidade Estadual Paulista - UNESP, Curso de Graduação em Fisioterapia, Presidente Prudente, SP. E-mail: Elaine_lozano@hotmail.com.

\section{RESUMO}

A população idosa vem aumentando progressivamente e a atuação fisioterapêutica deve acompanhar essa mudança para promover a saúde desses indivíduos. O equilíbrio pode ser comprometido com o envelhecimento, porém o exercício físico pode influenciar positivamente nesse declínio. O objetivo foi avaliar os benefícios da prática de exercício físico regular no equilíbrio de idosas ativas. Foi realizada avaliação antropométrica e a aplicação do Mini Exame do Estado Mental (MEEM) e da Escala de Equilíbrio de BERG frente em participantes de um programa regular de exercícios físicos, três vezes por semana com uma hora de duração. Os resultados obtidos foram médios de idade $71,5 \pm 6,5$ anos, IMC de $27,7 \pm 4,5 \mathrm{Kg} / \mathrm{m} 2$ para o ano de 2015 e MEEM $25,9 \pm 3,3$ pontos, sendo média do BERG foi de 53,67 $\pm 2,47$ pontos para 2011 e $53,27 \pm 4,95$ pontos para 2015. Os resultados não demonstraram significância estatística na melhora do equilíbrio, logo ocorreu manutenção dessa variável no período de quatro anos.

Palavras-chave: idoso, saúde, equilíbrio, exercício físico, envelhecimento.

\section{EFFECT OF REGULAR PHYSICAL EXERCISE IN THE ELDERLY LIVE BALANCE}

\section{ABSTRACT}

The elderly population is increasing gradually and physiotherapy performance must accompany this change to promote the health of these individuals. The balance can be compromised with aging, but exercise can positively influence this decline. The objective was to evaluate the benefits of regular physical exercise in balancing active women. It was conducted anthropometric measurements and the application of the Mini-Mental State Examination (MMSE) and the Berg Balance Scale forward in participating in a regular exercise program three times a week with an hour long. The results were average age $71.5 \pm 6.5$ years, BMI $27.7 \pm 4.5 \mathrm{~kg} / \mathrm{m} 2$ for the year 2015 and MMSE $25.9 \pm 3.3$ points, and the average was 53 BERG , $67 \pm 2.47$ points for 2011 and $53.27 \pm$ 4.95 points to 2015 . The results showed no statistically significant improvement in balance, so this variable was maintained in the four-year period.

Keywords: Elderly, health, balance, physical exercise, aging. 


\section{INTRODUÇÃO}

Nos dias atuais nos deparamos com elevado aumento da população idosa, isto torna um grande desafio para a sociedade quando direcionado ao serviço saúde, pois com o aumento da longevidade surge à necessidade de medidas que colaborem para que a população tenha uma vida mais saudável, por meio da prevenção de morbidade e mortalidade entre os idosos ${ }^{1}$.

A intervenção fisioterapêutica preventiva vem se tornando um importante aliado à promoção saúde, assim sendo, adaptar-se a um estilo de vida mais ativo permite ao indivíduo, redução da instalação de patologias, do risco de quedas e melhora do equilíbrio corporal ${ }^{2}$.

O equilíbrio corporal pode ser definido pela posição do corpo com um mínimo de oscilação na posição estática e durante atividades que causem desordem na orientação do corpo. Ele é dependente de um sincronismo entre as estruturas musculoesqueléticas, sistemas proprioceptivo, vestibular e visual ${ }^{3,4}$

O déficit de equilíbrio é uma das alterações que podem estar presentes no envelhecimento e merece uma atenção especial, podendo levar o idoso a uma predisposição ao risco de quedas e consequentemente a fraturas, que podem se agravar, levando a inúmeras complicações de saúde, até mesmo ao óbito ${ }^{5}$. Sendo assim a presença do déficit de equilíbrio pode vir a influenciar negativamente na qualidade de vida e interferir nas atividades de vida diária do idoso ${ }^{6}$.

Os sistemas visuais, vestibular, somato-sensorial e musculo-esquelético são responsáveis pela estabilidade do corpo humano, assim como a permanência do corpo no centro de gravidade quando estático. Com o envelhecimento ocorre a involução desses sistemas gradativamente, levando ao idoso diminuição da sua autonomia ${ }^{7}$.

A prática de exercício físico regular apresenta influência positiva no controle corporal, proporcionando melhora em todos os sistemas citados acima, responsáveis pelo controle postural $^{8}$.

Nesse contexto a avaliação do equilíbrio tornando-se um instrumento importante para elaboração de um tratamento preventivo específico, a fim de proporcionar uma melhor qualidade de vida e saúde ao idoso.

Diante do exposto, o presente estudo teve como objetivo avaliar o equilíbrio de idosas ativas participantes de um de um programa fisioterapêutico com enfoque em exercícios físicos regulares e supervisionados. 


\section{METODOLOGIA}

Estudo longitudinal de quatro anos com idosas inseridas em um programa fisioterapêutico preventivo para a terceira idade, junto ao Programa UNATI/UNESP de Presidente Prudente.

Todos os indivíduos foram comunicados previamente quanto ao objetivo e procedimentos do estudo e, após assinatura do termo de consentimento livre e esclarecido (TCLE), participaram de modo efetivo da pesquisa. O estudo foi aprovado pelo Comitê de Ética e Pesquisa da própria instituição (CAAE no 32860814.5.0000.5402)

Os critérios de elegibilidade elencados para a realização dos estudos estão dispostos a seguir:

\section{Critérios de Inclusão:}

1. Mulher com idade igual ou superior a 60 anos;

2. Frequentar o setor de Fisioterapia em Gerontologia da FCT/UNESP a pelo menos seis meses;

3. Estabilidade clínica e atestado médico, constatando que o mesmo está apto a realizar exercícios físicos moderados;

4. Ausência de diagnóstico médico de enfermidades que interferissem na prática de exercícios físicos (por exemplo, doenças cérebro-vasculares, cardíacas, pneumológicas, ortopédicas, reumáticas);

5. Aceitação à proposta de avaliação;

6. Assinatura do TCLE.

\section{Critérios de Exclusão:}

1. Indivíduo que havia realizado recentemente algum procedimento cirúrgico;

2. Participante que apresentasse algum tipo de intercorrência no decorrer do estudo;

3. Número de faltas igual ou superior a $25 \%$ no período.

4. Apresentar déficit cognitivo significativo após a aplicação do MEEM, sendo valor mínimo de 24 pontos.

O programa fisioterapêutico apresentado é composto pelos aspectos subseqüentes; alongamento, aquecimento, fortalecimento muscular, dinâmica e relaxamento. As sessões apresentaram frequência de três encontros por semana com uma hora de duração cada.

Inicialmente foi aplicado o Mini Exame do Estado Mental (MEEM); instrumento de inclusão/exclusão. Em seguida as participantes realizaram a avaliação antropométrica e a aplicação do teste de escala de Equilíbrio (BERG): 
a- MEEM - Teste que avalia a orientação temporoespacial, retenção, evocação, cálculo, linguagem/nomeação, e habilidades construtivas.

b- Avaliação antropométrica: peso, altura, índice de massa corporal (IMC).

c- Escala de equilíbrio Berg: A escala avalia o equilíbrio funcional em 14 itens comuns à vida diária. Cada um deles possui uma escala ordinal de cinco alternativas, que variam de 0 a 4 pontos, com pontuação máxima de 56.

Para análise dos dados foi utilizado o programa estatístico SPSS 17.0 for Windows ${ }^{\circledR}$. Foi realizado media e desvio padrão para idade e IMC. Para o teste de Berg, foi utilizado o teste de Wilcoxon, para amostras dependentes e dados não paramétricos. O valor de significância utilizado foi de $(p<0,05)$.

\section{RESULTADOS}

O estudo contou com a participação de 40 mulheres idosas; idade média de 71,51ะ6,56 anos; IMC de $27,70 \pm 4,57 \mathrm{Kg} / \mathrm{m} 2$ para o ano de 2015 . A pontuação média no MEEM foi $25,90 \pm 3,33$, dessa forma, não houve exclusão de nenhuma participante.

Quanto a variável equilíbrio avaliada por meio do teste de equilíbrio BERG, obteve-se

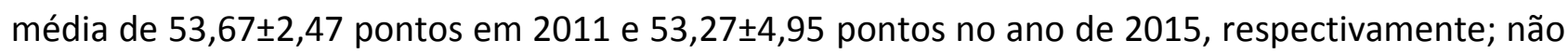
apresentando significância estatística.

\section{DISCUSSÃO}

No presente estudo num período de quatro anos, as participantes apresentaram aumento de IMC e redução de BERG sendo 27,37kg/ 53,55 pontos para 2011 e 27,70kg/53,27 para 2015.

Almeida et. al (2010) nos mostra que com aumento de peso ocorre concomitantemente a perda de massa muscular, consequentemente geram perda no equilíbrio dinâmico, visto que, o recrutamento das fibras musculares é maior para o equilíbrio dinâmico, quando comparado ao recrutamentos de fibras musculares para equilíbrio estático. Assim, acredita-se que mesmo não ocorrendo diferenças importantes para os valores de IMC e para o teste BERG durante o período de quatro anos, estes sejam índices importantes ${ }^{8}$.

Ao avaliar os participantes individualmente, foi observado aumento do equilíbrio em 40\%, redução de 40 \% e também a manutenção de $20 \%$ das idosas quando comparado com os dados de 2011 a 2015, o que nos mostra a eficácia do protocolo de exercício realizado, pois tanto o ganho, como a manutenção e ou a redução do declínio do equilíbrio corporal, ambos são objetivos do tratamento fisioterapêutico preventivo. 
Abreu e Caldas (2008) ao correlacionar velocidade da marcha, equilíbrio e idade, obteve como resultado a melhora do equilíbrio em idosas praticantes de um programa de exercício físico, quando comparado com idosas não praticantes ${ }^{9}$.

Na realizada por Oliveira e Prati (2014) composta por 14 idosos de ambos o sexo, ocorreu o aumento do escore para o teste Berg quando comparado a pré e pós-intervenção, sendo para homens incremento de 1,85 pontos e para mulheres incremento de 2,57 pontos. A pesquisa contou com um protocolo de exercícios fisioterapêuticos baseados em atividades para facilitação do equilíbrio por meio de trabalho de propriocepção ${ }^{10}$.

O estudo de Sá et. al (2012) cotou com uma amostra de 20 idosos, onde foi realizado uma intervenção de 18 semanas de atividades físicas com três sessões semanais e teve como resultado a diminuição de quedas antes e depois da intervenção. Esta diminuição mostra a eficácia da atividade física para o equilíbrio do idoso ${ }^{11}$. Bem como no estudo de SILVA et. al (2014) com 94 idosos submetidos a exercícios físicos regulares, sendo duas vezes por semanas durante seis meses, onde obtiveram melhora do equilíbrio, que por sua vem levam a melhora da qualidade de vida do idoso ${ }^{12}$.

\section{CONCLUSÃO}

Os resultados obtidos no presente estudo não demonstraram significância estatística na melhora do equilíbrio, logo ocorreu manutenção dessa variável no período de quatro anos.

\section{REFERÊNCIAS}

1. Vieira AAU, Aprile, MR, Paulino CA. Exercício Físico, Envelhecimento e Quedas em Idosos: Revisão Narrativa. Revista Equilíbrio Corporal e Saúde, 2015; 6(1):109-116. DOI:http://dx.doi.org/10.1590/S1809-98232013000100011.

2. Buranello MC, Campos SAO, Quemelo PV, Silva AVD. Equilíbrio corporal e risco de queda em idosas que praticam atividades físicas e sedentárias. Revista Brasileira de Ciências do Envelhecimento Humano, 2012; 8(3).

3. Maia DAR, Thomaz JQ, Kasse CA, Doná F. Efetividade da reabilitação vestibular na capacidade funcional de idosos com vestibulopatia. Revista Equilíbrio Corporal e Saúde, 2015; 5(2).

4. Silva Santos J, Valente JM, de Carvalho MA, de Magalhães Galvão K, Kasse CA. Identificação dos fatores de riscos de quedas em idosos e sua prevenção. Revista Equilíbrio Corporal e Saúde, 2015;5(2).

5. Castro PMMA, Magalhães AMD, Cruz ALC, Reis NSRD. Testes de equilíbrio e mobilidade funcional na predição e prevenção de riscos de quedas em idosos. Rev. bras. geriatr. Gerontol,2015; 18(1): 129-140. 
6. Antes DL, Wiest MJ, Mota CB, Corazza ST. Análise da estabilidade postural e propriocepção de idosas fisicamente ativas.Fisioterapia em Movimento.2014; 27(4).

7. Maciel ACC, Guerra RO. Prevalência e fatores associados ao defícit de equilibrio em idosos. R. bras. Ci e Mov, 2005; 13(1): 37-44.

8. Almeida APPV, Veras RP, Doimo LA. Avaliação do equilíbrio estático e dinâmico de idosas praticantes de hidroginástica e ginástica. Rev Bras Cineantropom Desempenho Hum,2010; 12(1): $55-61$.

9. Abreu SSE, Caldas CP. Velocidade de marcha, equilíbrio e idade: um estudo correlacional entre idosas praticantes e idosas não praticantes de um programa de exercícios terapêuticos. Rev Bras Fisioter, 2008; 12(4): 324-30.

10. Oliveira GG, Fernando AMP. Efetividade de um programa fisioterapêutico proprioceptivo para treino de equilíbrio em idosos institucionalizados. Revista Brasileira de Ciências do Envelhecimento Humano, 2014, 11(1).

11. Sá ACAM, Bachion MM, Menezes RLD. Physical exercises to prevent falls: a clinical trial with institutionalized elderly in the city of Goiânia in Brazil. Ciência \& Saúde Coletiva,2012; 17(8): 21172127

12. Silva JR, Camargo RCT, Nunes MM, Camargo TT, de Faria CRS, de Abreu LC. Análise da alteração do equilibrio, da marcha e o risco de queda em idosos participantes de um programa de fisioterapia. Revista e-ciência, 2015; 2(2). 